\title{
Microlens Enhancement in Respiratory Infection Diagnosis
}

\author{
Feiyue Teng ${ }^{1}$, Xinpei $\mathrm{Wu}^{1}$, Tseng-Ming $\mathrm{Chou}^{2}$ and Matthew Libera ${ }^{*}$ \\ 1. Dept. of Chemical Engr. \& Materials Science, Stevens Institute of Technology, Hoboken, NJ, USA. \\ 2. Laboratory for Multiscale Imaging, Stevens Institute of Technology, Hoboken, NJ, USA. \\ * Corresponding author: mlibera@stevens.edu
}

Molecular diagnostic sensors can rapidly identify the responsible pathogens of the infection and thus are critical to prescribe the most effective treatment. DNA/RNA microarrays comprise a miniaturized technology that enable us to simultaneously identify a large number of potentially infecting species to the level of an individual strain. They have been used extensively in molecular diagnosis because of the high automation and throughput. Many microarrays use fluorescence-based signaling technique. However, the arrays usually need to be viewed by sophisticated, high numerical aperture fluorescent microscope objective lenses, which are hard to integrate into portable point-of-care diagnostic devices. Instead of a high numerical aperture (NA) lens, a high-quality optical system can also be achieved by combining a microlens and a low NA lens to collect more light entering the optical microscope [1]. In another word, microlenses can be used to enhance the fluorescent signal of a microarray. Molecular beacons (MBs) are fluorescent self-reporting oligonucleotide probes, which have worked exceptionally well in a liquid assay. But when MBs are adapted to a microarray format, the proximity to an underlying hard surface can place conformational constraints on the MBs as well as raise the background signal via nonspecific interactions. Our previous work [2-4] has showed that tethering MB probes to highly swollen microgels patterned on solid substrates overcomes many of the conformational constraints imposed by a solid surface, because the diffuse interface between the aqueous medium and a hydrogel creates as water-like an environment as possible with maximal degrees of conformational freedom while still preserving the surface-location specificity associated with a microarray format.

We can furthermore amplify the fluorescent signal from the gel-tethered MBs by co-tethering dielectric microspheres, which act as spherical microlenses in immediate proximity to the emitter. Figure 1 shows streptavidin-functionalized PS microspheres (left) or silica microspheres (right) can be tethered by a directed self-assembly process to biotinylated PEG-based microgels patterned on silicon surface via electron beam lithography. The streptavidin-functionalized microspheres bind specifically to locations where there are biotinylated microgels. The number and spatial distribution of tethered microspheres can be controlled by the size of the microspheres and microgel patterning process. Decreasing gel pad size can isolate a single microsphere on a single gel pad consisted of several microgels (Figure 1 left). After microsphere tethering and blocking, biotinylated MB detection probes can be tethered to the remaining biotin sites on the microgels after the microgels are activated by streptavidin. We use a simplex assay based on Influenza A virus as an example of respiratory infection to study the lensing behavior. Figure 2 illustrates the signal generated when gel-tethered MBs are exposed to complementary targets. Figure 2 (a) and (d) indicate the signal generated from MBs tethered on microgels with different inter-gel spacing. Figure 2 (b) (c) (e) (f) shows that both PS and silica microsphere in immediate proximity to these MBs act as spherical microlenses to couple that fluorescent signal into the collecting objective lens of an optical microscope. Relative to equivalent hybridized MBs without microsphere, the lensing effect can increase the collected intensity by as much as ten times [5].

To conclude, we exploit the flexible and precise patterning capabilities of electron-beam lithography to 
create submicron-sized microgels of biotinylated PEG to direct the assembly of streptavidinfunctionalized PS/silica microspheres. Molecular-beacon detection probes are tethered to the same microgels. The microspheres can act as microlenses to enhance the fluorescent signal associated with geltethered MBs [6].

\section{References:}

[1] Y Ebenstein and LA Bentolila, Nature Nanotechnology 5 (2010), p. 99.

[2] X Dai et al., Soft matter 8 (2012), p. 3067.

[3] Y Ma et al., Analyst 142 (2016), p. 147.

[4] Y Ma, F Teng and M Libera, Analytical Chemistry 90 (2018), p. 6532.

[5] F Teng and M Libera, Langmuir: The ACS Journal of Surfaces and Colloids 34 (2018), p. 14969.

[6] This research project has been supported by the U.S. National Science Foundation via Grant Number CBET-1402706 and by the Army Research Office via Grant Number W911NF-17-1- 0332.
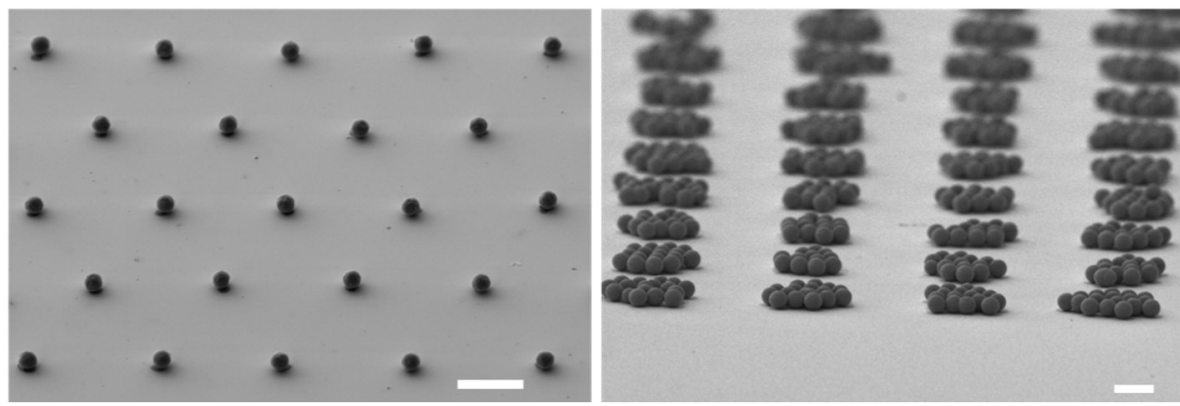

Figure 1. SEM images of microspheres assemble on continuous gel pad. The number of microspheres of each gel pad can be controlled by varying the size of the gel pad and microspheres. (a) $3 \mu \mathrm{m}$ PS microspheres on $1 \mu \mathrm{m}$ diameter gel pad. One microsphere per gel pad. (b) $5 \mu \mathrm{m}$ silica microspheres on 10 $\mu \mathrm{m}$ diameter gel pad. Scale bars $=10 \mu \mathrm{m}$.
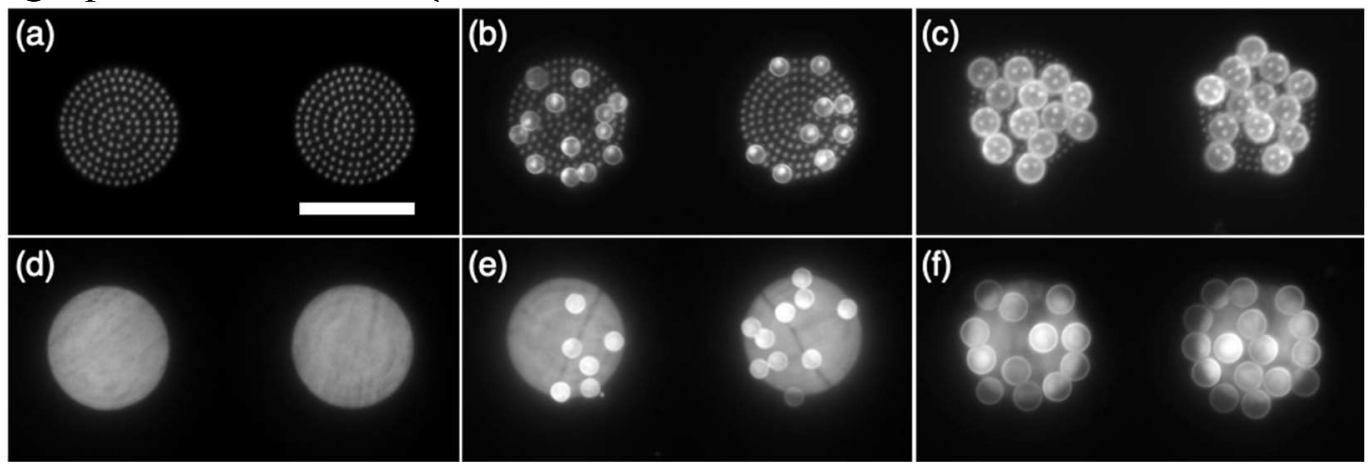

Figure 2. Fluorescent images of gel-tethered molecular beacon positive detection results with (b, c, e, f) or without (a,d) colloidal microlenses: (a) individual microgels. (b) $3 \mu \mathrm{m}$ PS microspheres on individual microgels. (c) $5 \mu \mathrm{m}$ silica microspheres on individual microgels. (d) continuous gel pads. (e) $3 \mu \mathrm{m}$ PS microspheres on continuous gel pads. (f) $5 \mu \mathrm{m}$ silica microspheres on continuous gel pads. Scale bar shows $20 \mu \mathrm{m}$. 